\title{
Potential immunotoxic effects of trichloroethylene-induced IV allergic reaction in renal impairment
}

\author{
JUN-FENG YU $U^{1}$, YAN-YAN FENG ${ }^{2}$, XIAO-FENG SHEN ${ }^{1}$ \\ ${ }^{1}$ Institute of Dermatology, The Fifth affiliated Hospital of Xinjiang Medical University, China \\ ${ }^{2}$ Institute of Dermatology, People's Hospital of Xinjiang Uygur Autonomous Region, China
}

\begin{abstract}
Trichloroethylene (TCE) is known to induce allergic contact dermatitis and subsequent occupational medicamentosa-like dermatitis (OMLD) with multi-system injuries, including liver, kidney, and skin injuries. However, the mechanisms underlying immune system dysfunction that result in organ injury have not yet been clearly elucidated. In the present study, we measured the levels of secreted cytokines by effect or T cells in TCE-treated guinea pigs to better understand the contribution of allergic disorders in renal injuries. We immunized guinea pigs with trichloroethylene using the Guinea Pig Maximization Test (GPMT) and scored the inflammation on the guinea pigs' skin. The kidney function and ultra-structural changes in the kidneys were detected using biochemical methods and electron microscopy. The deposition of cytokines was determined using immunohistochemistry. The sensitization rate was $63.16 \%$ in the TCE-sensitized groups. The electron microscopy results showed tubular epithelial cell mitochondrial swelling, vacuolar degeneration, and atrophy of the microvillus in the sensitized groups. A high degree of cytokine deposition was observed in the renal tubular proximal epithelial cells in the TCE-sensitized groups. As observed in this study, the variation in the level of immune system activation not only indicates that TCE can largely magnify the immune reaction but also suggests a potential role of immune dysfunction in renal impairment.
\end{abstract}

Key words: RT-PCR, trichloroethylene, renal injury.

(Centr Eur J Immunol 2017; 42 (2): 140-149)

\section{Introduction}

Trichloroethylene (TCE) has been widely used as an industrial solvent and a degreasing agent and has been considered a common occupational contaminant within the past twenty years $[1,2]$. Due to increased concern regarding the environment, the use of TCE has become less popular in the United States since the 1970s [3]. TCE was most comprehensively used in the coastal industrialized regions in China. Due to its wide commercial use, TCE has resulted in occupational poisoning. In addition, many serious TCE-induced exfoliative dermatitis cases have been reported, and some of the victims have even died. In addition to diseases such as severe dermatitis, TCE exposure has also been implicated in the development of various diseases, such as heart defects, cancers, systemic lupus erythematosus (SLE), and renal and liver dysfunction [4-6]. Severe skin lesions include exfoliative dermatitis, Steven-Johnson syndrome, and erythema multiforme. These skin disorders are also grouped under "Occupational medicamentosa-like dermatitis (OMLD) induced by TCE" according to the Chinese National Diagnostic Criteria. TCE-induced renal disorder has recently attracted increased attention. After long-term and high occupational exposure to trichloroethylene, increases in the renal cancer incidence have been ascribed to chronic damage of the renal tubule $[7,8]$. Increasing evidence has demonstrated that TCE is an animal carcinogen of renal tubule carcinomas in male rats with renal cell tumours [9]. Although trichloroethylene can induce obvious kidney damage, the impairment mechanism has not been well elucidated.

The immunotoxic effects of TCE to the skin and tissue are believed to be a result of allergic contact dermatitis. Thus, immune regulation dysfunction may play an important role in severe dermatitis and renal dysfunction. Moreover, it was reported that the immune reaction challenged by TCE is different from the IV allergic reaction induced by dinitrochlorobenzene (DNCB). In addition, it is difficult to induce severe viscera injury only by IV allergic reaction. [10] found that the level of sera IgE in mice increases after the ingestion of low doses of trichloroethylene in drink-

Correspondence: Xiao-Feng Shen, Institute of Dermatology, The Fifth affiliated Hospital of Xinjiang Medical University, China,

e-mail: 76897651@qq.com

Submitted: 28.03.2016; Accepted: 11.08.2016 
ing water. Moreover, several researchers have determined that the levels of $\operatorname{IgE}$ and $\mathrm{IgG}$ in the serum demonstrate a significant difference as a result of labour exposure to a low dose of trichloroethylene compared with the control group. Furthermore, the levels of immunoglobulin after exposure to a low concentration of TCE are lower compared to those measured after exposure to high concentrations of TCE [11]. Thus, these studies indicate that immune dysfunction may play an important role in TCE-induced renal impairment. A model using guinea pigs was developed to examine the relationship between TCE-induced immune system hyperactivity and renal injury. To better understand whether immune system dysfunction has a potent effect on kidney damage in an allergic contact dermatitis model, we measured the levels of cytokines in renal homogenates and detected the immune complex deposition in renal tissue. In addition, interferon $\gamma$ (IFN- $\gamma$ ), tumor necrosis factor $\alpha$ (TNF- $\alpha$ ), and interleukin 4 (IL-4) were selected as Th1 and Th2 cytokine indicators to evaluate the immune system hyperactivity.

\section{Material and methods}

\section{Animals}

Female guinea pigs weighing 250 to $300 \mathrm{~g}$ were purchased from Feidong Chang Lin He Animal Co., Ltd. (Anhui, China). All of the animals were healthy, housed in a plastic cage (six animals per cage), and maintained on a 12-h light/12-h dark cycle. The animals were provided tap water and a standard diet ad libitum. The healthy guinea pigs were randomly divided into four groups: blank control group, solvent (olive oil) control group, TCE group, and positive (DNCB) group. The experimental animal protocols were approved by the Animal Care and Use Committee of Anhui Medical University.

\section{Chemicals}

TCE and Freud's complete adjuvant (FCA) were purchased from Sigma (St. Louis, MO, USA), and olive oil (vehicle for TCE) was provided by Shanghai Chemical Reagent Company. Anti-TNF- $\alpha$, anti-IL-4, and anti-IFN- $\gamma$ antibodies were provided by Uscnlife (Wu Han, China). TNF- $\alpha$, IL-4, and IFN- $\gamma$ ELISA assay kits were purchased from the Rapidbio Limited Company (CA, USA). The RTPCR protocols were provided by Fermentas Bioscience Limited Company. Trizol reagent was purchased from Invitrogen Company (California, USA).

\section{Guinea pigs maximisation test}

The GPMT methods of allergic contact dermatitis were performed as previously described [12]. A schematic of the experiment is shown in Fig. 1. The allergenic reactions on the animal's skin were scored using a 4-point scale: 0 , no reaction; 1 , scattered mild redness; 2 , moderate and dif- fuse redness; and 3, intensive erythema and swelling. The sensitisation frequency of the TCE groups was calculated according to the allergic reaction scores.

\section{Histopathological and ultra-structure observation in the kidney}

On the $15^{\text {th }}$ day, the TCE-sensitized animals were divided into sensitized-positive (+) and -negative (-) groups. The animals were sacrificed by $\mathrm{CO}_{2}$ asphyxiation on the $15^{\text {th }}$ and $17^{\text {th }}$ days, and the animals in the other three groups were sacrificed on the $15^{\text {th }}$ day. The kidney tissue was fixed in $0.1 \mathrm{~mol} / \mathrm{l}$ glutaraldehyde-paraformaldehyde solution for $12 \mathrm{~h}$ and observed using an electron microscope. The other tissues were fixed in $10 \%(V / V)$ formalin, embedded in paraffin, stained with haematoxylin and eosin, and observed using light microscopy.

\section{Evaluation of renal function}

The renal function detection was performed as previously described [12].

\section{Cytokine secretion level assessment in kidney tissue homogenates using ELISA}

Before the animals were sacrificed by $\mathrm{CO}_{2}$ asphyxiation, blood was collected by cardiac puncture and then centrifuged at $3000 \mathrm{rpm}$ and $4^{\circ} \mathrm{C}$ for $15 \mathrm{~min}$. The washed kidneys were wrapped with absorbent papers and weighed. The tissue was placed in a glass culture dish on ice and sheared with ophthalmic scissors. A $10 \%$ renal homogenate was prepared in $0.1 \mathrm{mM}$ PBS ( $\mathrm{pH} 7.4$ ) in a glass homogeniser, and the homogenate was subsequently centrifuged at $2000 \times g$ and $4^{\circ} \mathrm{C}$ for $10 \mathrm{~min}$.

The TNF- $\alpha$, IL-4, and IFN- $\gamma$ levels in the peripheral blood and tissue homogenate were quantified using com-

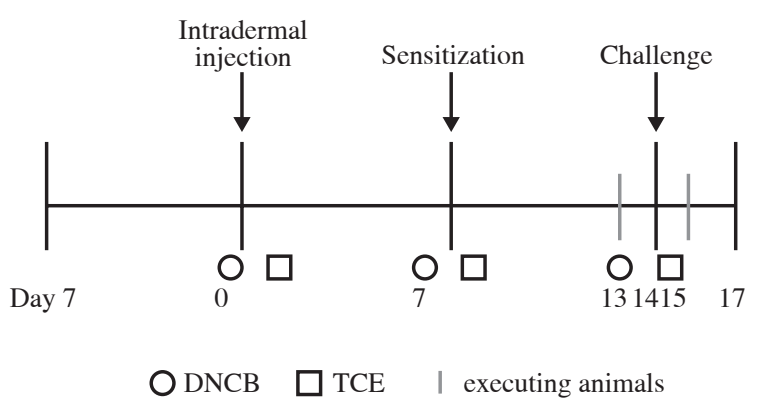

Fig. 1. Experimental schedule for the induction of GPMT. After 7 days of adaptive feeding, DNCB and TCE were applied to the back of animals by intradermal injection. After 1 week, DNCB and TCE were topically applied on the back of guinea pig for sensitization. After 2 weeks, animals were challenged with DNCB and TCE. Executing animals and taking materials at $13^{\text {th }}$ day, $15^{\text {th }}$ day and $17^{\text {th }}$ day 
mercially available ELISA kits (Rapidbio Co.) according to the manufacturer's instructions. Briefly, the renal tissue homogenate was incubated with anti-guinea pig IgG-horseradish peroxidase-conjugated plates for $1 \mathrm{~h}$ at room temperature. After the plates were washed three times with $1 \%$ Tween 20 in PBS, tetramethylbenzidine A and B solution was added, and the plates were incubated for $15 \mathrm{~min}$ at room temperature. The reaction was terminated by the addition of $50 \mu \mathrm{l}$ of $\mathrm{H}_{2} \mathrm{SO}_{4}$ solution. The colour development was measured at $450 \mathrm{~nm}$ using a microplate reader.

\section{Immunohistochemistry assessment of TNF- $\alpha$, IL-4, and IFN- $\gamma$}

The sections were deparaffinized in xylene and rehydrated in a series of graded alcohols. We then detected the precipitation of TNF- $\alpha$, IL- 4 , and IFN- $\gamma$ in all groups of renal tissue. A series of immunoreaction depositions in the renal tubule was performed based on a subjective semi-quantitative assessment of the deposition intensity. The immuno-histochemistry-based staining used to assess the cytokine deposition intensity was performed as previously described [12]. The concentrations of monoclonal antibodies diluted with $0.1 \mathrm{mM}$ PBS (pH 7.4) were as follows: $1: 500$ (TNF- $\alpha$ ), $1: 400$ (IL-4), and $1: 300$ (IFN- $\gamma$ ). The biopsy scores were estimated by three pathology professors.

\section{Isolation of RNA from renal tissue and RT-PCR analysis of TNF- $\alpha$ and IL-4 mRNA levels}

The RNA was extracted using the Trizol reagent (Invitrogen, USA) according to the manufacturer's protocol with some minor modifications. Briefly, the tissue was homogenized in PBS buffer solution and then partially clarified by centrifugation at $2000 \mathrm{rpm}$. Then, $250 \mu \mathrm{l}$ of the supernatant was transferred into a new tube containing $750 \mu \mathrm{l}$ of Trizol reagent, and the mixture was incubated for $5 \mathrm{~min}$ at room temperature. Then, $200 \mu \mathrm{l}$ of chloroform was added to the solution, and the mixture was vortexed for $30 \mathrm{~s}$, incubated at room temperature for $3 \mathrm{~min}$, and centrifuged at 12,000 $\mathrm{rpm}$ and $4^{\circ} \mathrm{C}$ for $15 \mathrm{~min}$. The RNA was precipitated by the addition of $500 \mu \mathrm{l}$ of ice-cold propan-2-ol, mixed, and centrifuged for $15 \mathrm{~min}$. The RNA pellet was washed with 1000 $\mu \mathrm{l}$ of $75 \%$ (v/v) ethanol/water and centrifuged at $7000 \mathrm{rpm}$. The supernatant was decanted, and the pellet was air-dried and then resuspended in $25 \mu \mathrm{l}$ of diethylenepyrocarbonate-treated water. The RNA concentration and purity were determined using spectrophotometry and gel analysis, and the RNA was then stored at $-85^{\circ} \mathrm{C}$.

The reverse transcription reaction of RNA and PCR were performed according to the manufacturer's instructions. Three sets of primer sequences for TNF- $\alpha$, IL-4, and $\beta$-actin were used for polymerase chain reaction as follows. The upstream primer sequence of used for TNF- $\alpha$ was 5'-ttcacaccacacctttccac-3', and the downstream primer sequence was 5'- cttggctctcagtcctcttca-3'. The upstream and downstream primer sequences for IL-4 were 5' -ctgaagagggtgaagggttatg-3' and 5' -cttgggttgtgaaacgaatgtt-3', respectively, and the $\beta$-actin primer sequences were 5'-atcactgccacccagaagact-3' and 5'-ctgtagccgaactcattgtca-3'. The difference in the levels of cytokine expression in the groups was evaluated using the TNF- $\alpha / \beta$-actin and IL- $4 / \beta$ actin ratios.

\section{Statistical analysis}

All of the statistical analyses were performed using SPSS version 12.0 software. The results were expressed as the mean values \pm standard error of the mean (S.E.M.). The statistical analysis of the data score was performed by analysis of variance (ANOVA) followed by the least significant difference test (LSD). A p-value of less than 0.05 was considered to be statistically significant.

\section{Results}

\section{Sensitisation rate of guinea pigs with TCE treatment}

The skin lesion scores and sensitisation rate of the GPMT challenged by TCE or DNCB were calculated using the following formula: mean scores $=$ (score of redness + score of swelling)/number of animals in the group. Based on the allergic reaction score of the skin, the sensitisation rate of TCE-treated guinea pigs was $63.16 \%$, whereas that of the DNCB-positive control group was $100 \%$.

\section{Histopathological and cellular structural changes in TCE-treated GPMT}

Five-micrometer-thick paraffin slides of renal tissue were stained with hematoxylin and eosin (H\&E) and analyzed through light microscopy. Representative images from the slides were imaged. No significant histopathological changes were observed, and no neutrophil infiltration was observed in the blank and solvent control groups. However, there was significant neutrophil infiltration in the TCE-sensitized and DNCB groups. These results are shown in Fig. 2.

For the electron microscopic examination, necrosis of the villi and microvilli of the brush border of renal tubular epithelial cells, as well as shortened and swollen residual microvillus, were observed in the TCE-sensitized group on the $15^{\text {th }}$ day (Fig. 3). Vacuolar degeneration of the mitochondria was observed, and the number of tubular epithelial cells decreased, as assessed through electron microscopy. However, no significant morphological changes were determined in the blank control and solvent control groups. In the TCE-sensitized group (on the $17^{\text {th }}$ day), the same pathological changes were observed in the renal tubular epithelial cells: swelling, vacuolar degeneration of the mi- 

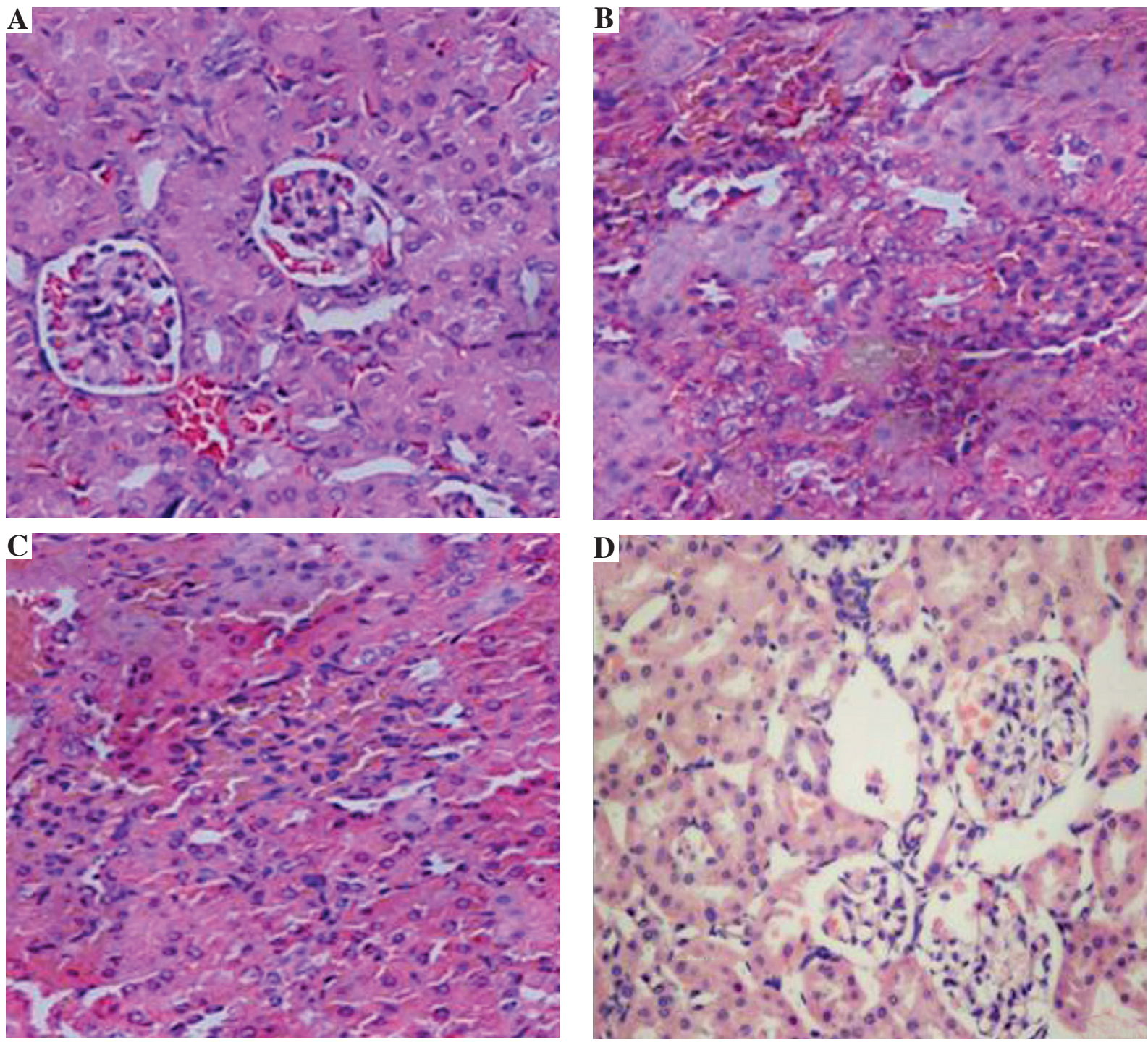

Fig. 2. Representative micrographs of renal histopathological sections from guinea pigs treated with trichloroethylene, stained with hematoxylin-eosin. A) Control group; B) TCE sensitized $15^{\text {th }}$ day group; C) TCE sensitized $17^{\text {th }}$ day, the section show infiltrations of neutrophil; D) TCE negative group

tochondria, microvilli shrinkage, and disappearance of the brush border. With the exception of a slight change in the foot process and glomerular basement membrane, there was no obvious difference in the TCE-sensitized groups.

\section{Effects of TCE treatment on kidney function}

The contents of BUN and $\mathrm{Cr}$ did not demonstrate a significant difference between the solvent control and blank control groups $(p>0.05$, Table 1$)$. However, the levels of BUN and $\mathrm{Cr}$ were increased significantly $(p<0.05)$ in the TCE-sensitized group on the $15^{\text {th }}$ and $17^{\text {th }}$ days compared with the solvent group, and the level of BUN was increased significantly in the DNCB group. However, no significant difference was observed in the $\mathrm{Cr}$ levels. The levels of BUN showed a significant difference between the TCE-sensitized groups and the TCE-sensitized group on the $13^{\text {th }}$ day control group $(p<0.05)$. In addition, a significant difference was detected between the TCE-sensitized groups and TCE-negative groups.

\section{Detection of cytokine contents in renal tissue homogenates}

The mean cytokine levels in the renal homogenates are summarized in Table 1. The levels of cytokines had no significant difference between the solvent control group and the blank control group $(p>0.05$, Table 1$)$. However, 

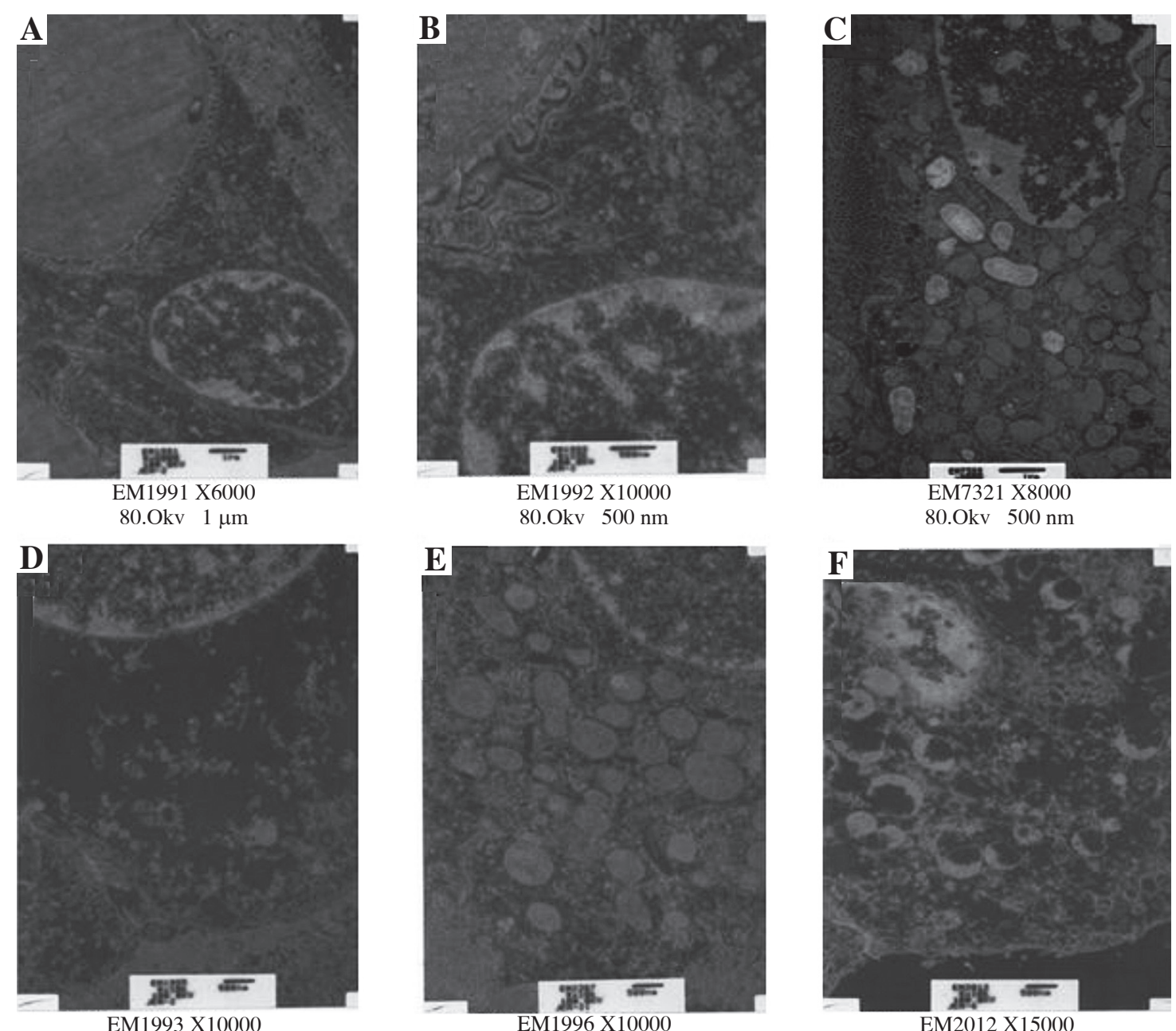

EM1992 X10000 $80 . \mathrm{Okv} 500 \mathrm{~nm}$
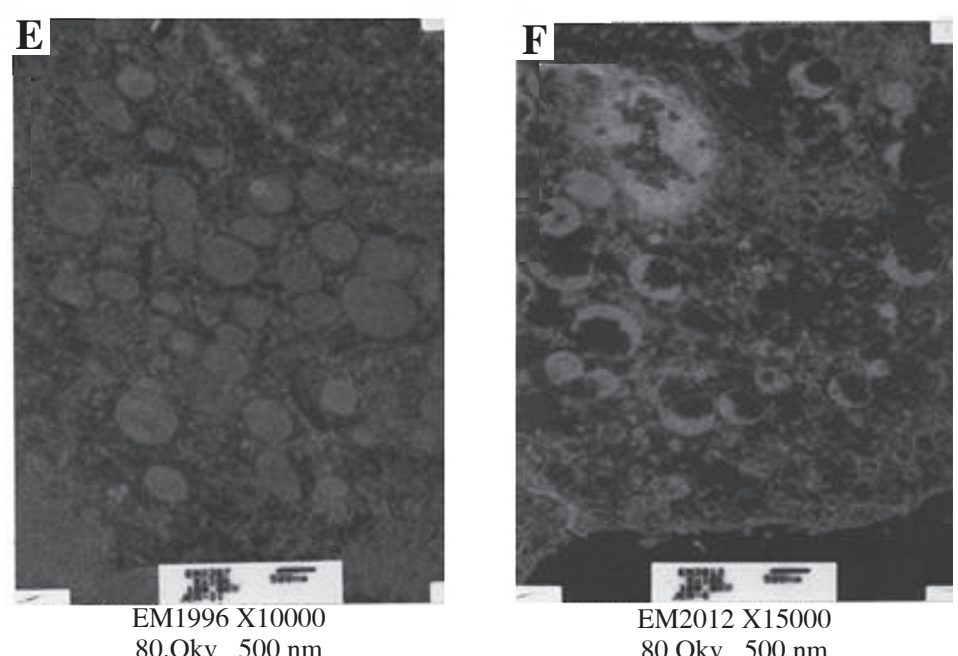

EM1993 X10000
80.0kv $500 \mathrm{~nm}$

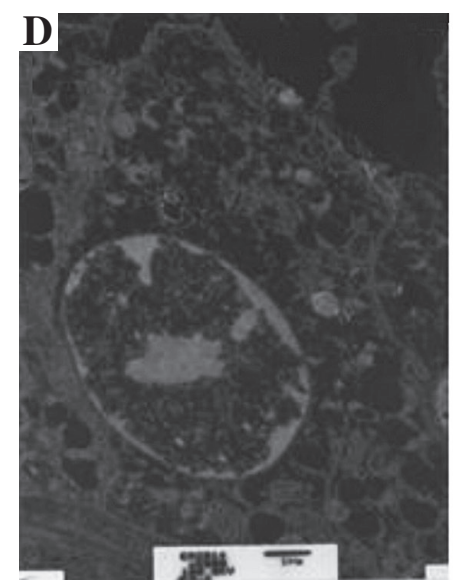

EM2014 X6000

$80 . \mathrm{Okv} 1 \mu \mathrm{m}$

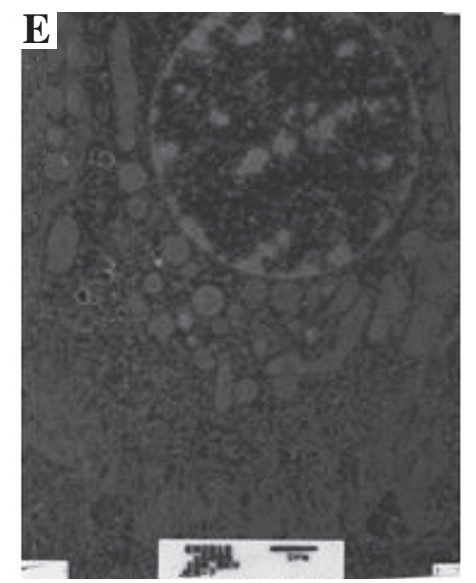

EM2005 X6000

$80 . \mathrm{Okv} 1 \mu \mathrm{m}$

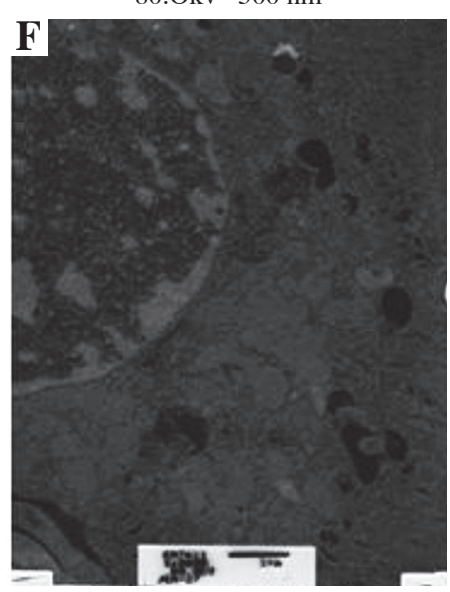

EM7417 X10000 $80 . \mathrm{Okv} 500 \mathrm{~nm}$

Fig. 3. Ultrastructure changes of renal glomerular and proximal tubules cells after trichloroethylene challenged by electron microscope. A) Blank control group, no obvious change of glomerular. B) Solvent control group. C) TCE sensitized $13^{\text {th }}$ day group, few vacuolar degeneration of renal tubular cells' mitochondria. D) TCE sensitized $15^{\text {th }}$ day, the necrosis and rupture of villi and microvilli of brush border within renal tubular epithelial cells and decrease of mitochondria. E) TCE negative $15^{\text {th }}$ day, swelling of the mitochondria within renal tubular epithelial cells. F, G) TCE sensitized $17^{\text {th }}$ day, obvious swelling and vacuolar degeneration of the mitochondria. H) TCE negative $17^{\text {th }}$ day, swelling of the mitochondria. I) DNCB sensitized $17^{\text {th }}$ day, slight vacuolar degenation of the mitochondria 
Table 1. Renal function and cytokines contents of renal homogenate in guinea pigs after GPMT challenged with TCE

\begin{tabular}{lcccccc}
\hline Group & Numbers & BUN $(\mathbf{m g} / \mathbf{m l})$ & $\mathbf{C r}(\boldsymbol{\mu m o l} / \mathbf{l})$ & $\mathbf{I L - 4}(\mathbf{p g} / \mathbf{m l})$ & $\mathbf{I F N}-\gamma(\mathbf{p g} / \mathbf{m l})$ & TNF- $\alpha(\mathbf{p g} / \mathbf{m l})$ \\
\hline Blank & 5 & $11.3 \pm 1.4$ & $21.2 \pm 3.1$ & $22.44 \pm 3.34$ & $48.54 \pm 3.82$ & $30.64 \pm 4.12$ \\
\hline Solvent control & 5 & $11.1 \pm 1.3$ & $21.4 \pm 1.7$ & $23.02 \pm 3.64$ & $46.53 \pm 4.47$ & $30.15 \pm 3.48$ \\
\hline TCE $13^{\text {th }}$ d & 5 & $12.6 \pm 1.5$ & $22.7 \pm 2.5$ & $26.50 \pm 3.75^{\bullet}$ & $50.75 \pm 4.25$ & $33.54 \pm 3.12$ \\
\hline TCE sensitized $15^{\text {th }}$ day & 9 & $15.7 \pm 1.2^{\bullet *}$ & $24.8 \pm 1.9^{\bullet *}$ & $28.48 \pm 2.85^{* \bullet}$ & $59.45 \pm 7.63^{* \bullet}$ & $37.77 \pm 4.41^{* \bullet}$ \\
\hline TCE negative $15^{\text {th }}$ day & 4 & $11.6 \pm 1.9$ & $19.3 \pm 3.4$ & $23.54 \pm 3.36$ & $51.24 \pm 7.12$ & $32.74 \pm 2.82$ \\
\hline TCE sensitized $17^{\text {th }}$ day & 8 & $17.4 \pm 2.6^{\bullet *}$ & $25.8 \pm 2.5^{\bullet *}$ & $27.39 \pm 2.14^{\bullet}$ & $61.22 \pm 7.54^{* *}$ & $42.48 \pm 3.94^{* \bullet}$ \\
\hline TCE negative $17^{\text {th }}$ day & 5 & $14.7 \pm 1.2^{*}$ & $21.5 \pm 2.4$ & $25.88 \pm 3.37$ & $53.65 \pm 6.85^{*}$ & $28.06 \pm 2.92$ \\
\hline DNCB group & 6 & $12.14 \pm 1.15$ & $19.20 \pm 1.50$ & $25.25 \pm 3.50$ & $50.26 \pm 4.75$ & $31.66 \pm 3.45$ \\
\hline F & - & 9.4 & 4.34 & 3.12 & 12.38 & 8.52 \\
\hline$p$ & - & 0.000 & 0.014 & 0.020 & 0.000 & 0.000 \\
\hline
\end{tabular}

${ }^{\circ} p<0.05$, compared with solvent control; ${ }^{\triangle} p<0.05$, compared with TCE1 $3^{\text {th }} d$ group: ${ }^{*} p<0.05$, compared with corresponding TCE negative group

Table 2. Deposition of cytokines on the renal paraffin sections in animals challenged with TCE

\begin{tabular}{lcccc}
\hline Group & Numbers & IL-4 & IFN- $\gamma$ & TNF- $\alpha$ \\
\hline Blank & 5 & $0.8 \pm 0.2$ & $1.4 \pm 0.6$ & $1.6 \pm 0.5$ \\
\hline Solvent control & 5 & $1.0 \pm 0.4$ & $1.2 \pm 0.4$ & $1.4 \pm 0.2$ \\
\hline TCE sensitized $15^{\text {th }}$ day & 5 & $2.8 \pm 1.2^{* *}$ & $3.6 \pm 1.4^{*}$ & $4.2 \pm 1.2^{* *}$ \\
\hline TCE negative $15^{\text {th }}$ day & 4 & $1.4 \pm 0.8$ & $2.0 \pm 0.8$ & $2.5 \pm 0.2$ \\
\hline TCE sensitized $17^{\text {th }}$ day & 5 & $3.2 \pm 1.6^{*}$ & $4.2 \pm 2.3^{*}$ & $4.6 \pm 2.0^{*}$ \\
\hline TCE negative $17^{\text {th }}$ day & 5 & $2.0 \pm 1.4$ & $2.6 \pm 1.2^{*}$ & $3.2 \pm 0.4^{*}$ \\
\hline$p$ & -- & 0.018 & 0.000 & 0.002 \\
\hline${ }^{*} p<0.05$, compared with solvent control: $^{*} p<0.05$, compared with corresponding TCE negative group &
\end{tabular}

the levels of TNF- $\alpha$, IL-4, and IFN- $\gamma$ showed a significant difference between the TCE-sensitized guinea pigs groups and the solvent control group. Moreover, there was a significant difference in TNF- $\alpha$ and IFN- $\gamma$ levels between the TCE-sensitized guinea pig groups and the TCE-unsensitized group. However, no difference in the IL-4 content was detected between the TCE-sensitized group on the $17^{\text {th }}$ day and the no unsensitized group.

\section{Immunohistochemistry-based cytokine detection in renal tissue}

The mean scores of the deposition of TNF- $\alpha$, IL- 4 , and IFN- $\gamma$ in all of the groups are shown in Table 2. Representative micrographs are shown in Fig. 4. There were no immune complex depositions in the blank control and solvent control groups. In addition, there were no differences in the cytokine depositions between the TCE dermatitis (-) groups, but a significant difference was observed between the TCE-sensitized group on the $15^{\text {th }}$ and $17^{\text {th }}$ days and the solvent control group $(p<0.05)$. Moreover, there was a significant difference in the TCE-sensitized guinea pig groups compared with the TCE-unsensitized group. Based on the immunohistochemistry images, we determined that the immune complex was deposited on the tubular epithelial cells, particularly along the cortical proximal tubular cells.

\section{Expression of TNF- $\alpha$ and IL-4 mRNA in renal tissue}

We performed an RT-PCR assay to detect the cytokine mRNA levels in the renal tissue samples from all of the animal groups. The mean ratios of TNF- $\alpha / \beta$-actin and IL- $4 / \beta$-actin are shown in Table 3 , and representative micrographs are shown in Fig. 5. According to the ratio, the expression level of IL-4 mRNA showed a clear difference between the solvent control group and the TCE-sensitized guinea pig group. The expression of IL-4 mRNA in the TCE-sensitized group was higher compared with that found in the solvent control group. Moreover, TNF- $\alpha$ was also detected at nearly the same expression level as IL-4.

\section{Discussion}

Trichloroethylene (TCE) has long been known to be a major chemical hazard associated with occupational expo- 

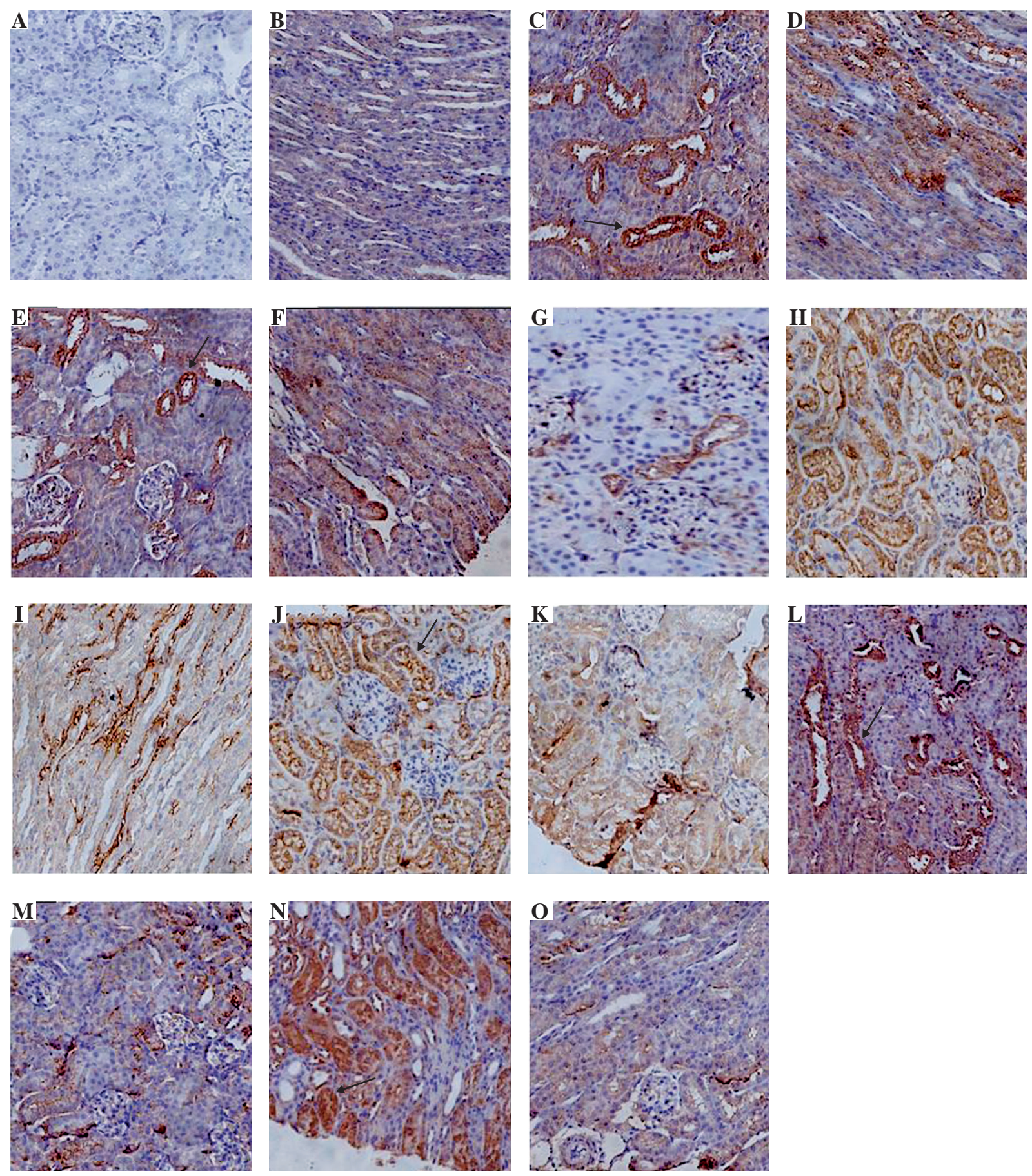

Fig. 4. Precipitation of cytokines immune complex on renal tissue using immunohistochemistry detected with $D A B$ kits. A) Negative control group. B) Solvent control group, no deposition of immune complex. C) TCE sensitized15 $5^{\text {th }}$ day, it was shown that high concentration of IL-4 were deposited along with renal proximal tubular epithelial cells, and no deposition of immune complex of renal glomeruli. D) TCE negative $15^{\text {th }}$ day, slight deposition of IL-4 complex. E) TCE sensitized $17^{\text {th }}$ day, the similar precipitation intensity with TCE sensitized $15^{\text {th }}$ day. F) TCE negative $17^{\text {th }}$ day. G-K) The deposition of cytokine TNF- $\alpha$, the representative micrographs of $(\mathrm{H})$ and $(\mathrm{J})$ were TCE sensitized group, black arrow showed immunoreactant deposition of cytokines with renal proximal tubular epithelial cells. G) Solvent control group. L-O) The deposition of cytokine IFN- $\gamma$, the micrographs of (L) and (N) were TCE sensitized groups, the others were TCE negative groups 
Table 3. The expression levels of TNF- $\alpha$ and IL-4mRNA in renal tissue challenged with TCE

\begin{tabular}{lccc}
\hline Group & Numbers & TNF- $\alpha / \beta$-action & IL-4/ $\beta$-action \\
\hline Blank & 3 & $0.4 \pm 0.2$ & $0.3 \pm 0.2$ \\
\hline Solvent control & 3 & $0.4 \pm 0.2$ & $0.4 \pm 0.4$ \\
\hline TCE sensitized $15^{\text {th }}$ day & 4 & $1.2 \pm 0.3^{* \bullet}$ & $1.4 \pm 0.5^{*}$ \\
\hline TCE negative $15^{\text {th }}$ day & 4 & $0.6 \pm 0.2$ & $0.4 \pm 0.2$ \\
\hline TCE sensitized $17^{\text {th }}$ day & 4 & $1.0 \pm 0.2^{* \bullet}$ & $1.2 \pm 0.4^{*}$ \\
\hline TCE negative $17^{\text {th }}$ day & 3 & $0.6 \pm 0.4$ & $0.4 \pm 0.2^{*}$ \\
\hline$p$ & - & 0.038 & 0.026
\end{tabular}
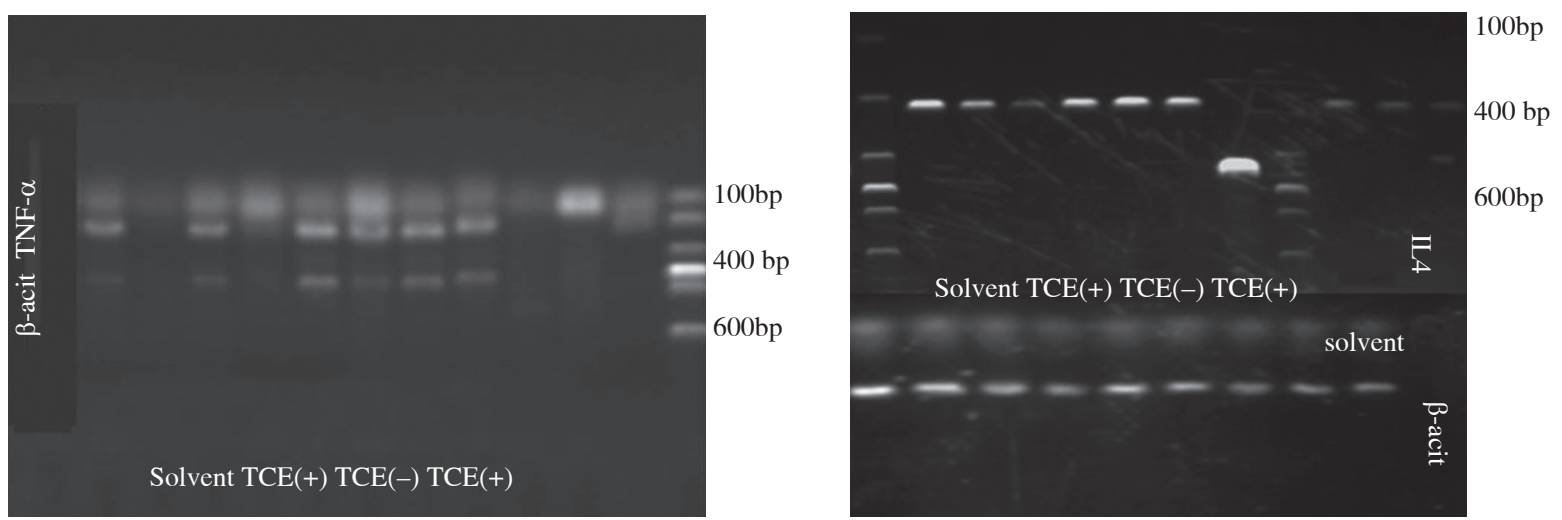

Fig. 5. The expression levels of TNF- $\alpha$ and IL-4mRNA. Higher expression of TNF- $\alpha$ and IL-4mRNA in TCE sensitized group compared with solvent control group and TCE negative group. The difference had statistically significant in TCE treated groups

sure due to its extensive use in industry as a dry-cleaning and metal-degreasing agent. Exposure to TCE can induce significant skin lesions and multi-system impairment. There have been several reports of TCE-related dermatitis in the southern areas of China. Moreover, a number of death cases, which have been primarily ascribed to serious organ dysfunction after long-term and high-concentration TCE exposure, have occurred. Recently, TCE-induced renal disorder has drawn considerable attention. It has been widely established that IV allergic reactions play an important role in DMLT [13] found that lymphocyte infiltration was apparent in the kidneys of female $\mathrm{MRL}^{+/+}$mice that were chronically exposed to TCE via their drinking water for 48 weeks. Jia et al. [14] found that cytokine overexpression, such as the overexpression of IL-1 and chemokines, plays an important role in trichloroethylene-induced hypersensitivity dermatitis. Furthermore, Blossom et al. [15] found that long-term exposure to TCE in vivo can activate $\mathrm{CD}^{+}$effect or $\mathrm{T}$ cells and induce neutrophil infiltration and inflammation. Moreover, Blossom et al. [16], found that developmental and early life TCE exposure can modulate immune function and increase $\mathrm{CD} 4^{+} \mathrm{T}$ cell production of the proinflammatory cytokine IFN- $\gamma$ in autoimmune-prone $\mathrm{MRL}^{+/+}$mice. Wang also found that splenocytes from mice treated with TCE for 24 weeks secreted significantly higher levels of IL-17 and IL-21 compared to splenocytes from control groups. The increased release of these cytokines showed a dose-dependent response [17, 18] also found that the level of IL-10 was statistically significant in workers exposed to $<12 \mathrm{ppm}$ and in workers exposed to $\geq 12 \mathrm{ppm}$ of TCE compared with unexposed workers. Zhang et al. [19] found that the levels of $\mathrm{CD}^{+}$ and $\mathrm{CD}^{+} \mathrm{T}$ cells and the ratio of $\mathrm{CD} 4^{+} / \mathrm{CD}^{+} \mathrm{T}$ cells were increased in the earlier phase of OMLD. Taken together, these studies suggest that TCE can induce immune system dysfunction in allergic contact dermatitis. However, to determine whether immune system dysfunction contributes to the mechanism of renal impairment in TCE-induced allergic reaction, we detected the mRNA expression levels of cytokines in renal tissue.

In our study, GPMT, which is considered a standard method, was performed to study the mechanism of con- 
tact hyper dermatitis. The sensitization rate of TCE was found to be $63.16 \%$ in the present study. Serious oedema and erythema were observed in the skin of the sensitized guinea pigs. These results indicate that TCE can induce skin lesions by IV allergic reaction. Moreover, the renal function was significantly different in the TCE-sensitized animal groups. The levels of BUN and $\mathrm{Cr}$ were increased significantly in the TCE-sensitized groups on the $15^{\text {th }}$ and $17^{\text {th }}$ days compared with the solvent group $(p<0.05)$. Using light microscopy and electron microscopy, we detected renal injury, including inflammatory cell infiltration, segmental basement membrane thickening, and vacuolar degeneration of the mitochondria in renal tubular epithelial cells, and found that the renal tubular damage was more severe. These results show that the skin and kidney were injured in the TCE-sensitized groups. In addition, we detected the levels of cytokines secreted by $\mathrm{CD}^{+} \mathrm{T}$ cells in renal tissue to study the effects of immune system dysfunction on kidney impairment. An increasing body of evidence suggests that effector $\mathrm{T}$ cell-mediated reactions play a role in the pathogenesis of renal impairment. In our previous study, we found that TCE results in not only obvious skin injury with IV allergic contact dermatitis but also high levels of cytokines in the serum, which amplify the inflammatory immunoreaction. It is well known that different effector subsets of $\mathrm{CD}^{+} \mathrm{T}$ cells have been described as $\mathrm{CD}^{+} \mathrm{T}$ type 1 cells (Th1), Th2, Th17, and regulatory $\mathrm{CD}^{+} \mathrm{T}$ cells, which play important roles in the inflammatory response [20]. In particular, the dysfunction of Th1 and Th2 cells is of widespread concern in an allergic reaction. The production of IFN- $\gamma$ and TNF- $\alpha$ by Th1 cells is thought to be involved in governing cellular immunity against intracellular pathogens, whereas Th2 cell production of IL-4, IL-5, IL-10 is thought to enhance immune responses that protect against extracellular pathogens [21]. The characteristics of TCE-induced hypersensitivity dermatitis were presumably determined by dysregulation of T-cell mediated immune mechanisms in which soluble mediators such as pro-inflammatory cytokines and chemokines play a crucial role. Some studies have shown an increased expression of TNF- $\alpha$, IFN- $\gamma$ and IL-6 in both skin biopsies and peripheral $\mathrm{CD}^{+} \mathrm{T}$ cells in patients with drug-induced maculopapular exanthema (MPE) [22, 23]. TCE could increase the mean percentage of central memory CD4+ T cells and expand the populations of the effector memory $\mathrm{T}$ cell subset in mice, which might contribute to uncontrolled inflammation leading to tissue pathology. Some studies have found that TCE exposed mice show more variation in IFN- $\gamma$ and IL-17 than control groups. This result might suggest that in certain mice within the TCE treatment groups, the $\mathrm{CD} 4^{+} \mathrm{T}$ cells may have converted to from a naive to memory to a proinflammatory phenotype. In a previous study, we found that the serum levels of cytokines, including IL-10, TNF- $\alpha$,
IgG, IgM, and IgG-CIC, are increased significantly in the TCE-sensitized groups [24]. To further evaluate the effects of TCE on Th1/Th2 cytokine secretion, we measured the levels of cytokines in renal tissue homogenates and detected the immune complex precipitation in renal tissue, and the results are consistent with the concept of immune system allergic disorders. Consequently, IFN- $\gamma$, TNF- $\alpha$, and IL- 4 were selected as Th1 and Th 2 cytokine indicators. Our present results show that the levels of TNF- $\alpha$, IL-4, and IFN- $\gamma$ demonstrated a significant difference between TCE-sensitized guinea pigs and the solvent control group. In addition, there was a significant difference in the TNF- $\alpha$ and IFN- $\gamma$ levels between the TCE-sensitized guinea pigs and the negative control group. The deposition of cytokines was mainly located in renal proximal tubular cells, and there was no immune complex deposition in the renal glomerulus. In addition, we assessed the mRNA expression levels of the cytokines in renal tissue using RT-PCR and found the some secreted cytokines contributed to the inflammatory reaction. The expression of IL- 4 and TNF- $\alpha$ mRNA in the TCE-sensitized group was higher compared with that found in the solvent control group. The RT-PCR results suggest that the cytokine secretion function of renal tubular cells is enhanced after irritation by inflammatory cytokines. Our results suggest that TCE induced pro-inflammatory responses and causes an imbalance in Th1/Th2-type helper T-cells. Taken together, the findings show that TCE and its main metabolites DCVC can induce immune system regulation functional disturbances and that subsequently higher levels of cytokines may comprehensively magnify the inflammatory reaction. Thus, TCE not only induces T cell-mediated allergic reactions but also results in renal impairment by amplifying the immune reaction. However, the mechanisms associated with the activation of effect or $\mathrm{T}$ cells underlying immune system dysfunction and renal injury-related cytokines require further comprehensive investigations. In our ongoing study, the study of signal transduction pathways, the activation of the transcription factor NF- $\kappa \mathrm{B}$, and the activation of the effector $\mathrm{T}$ cells will be further explored.

To summarise, this study evaluated TCE-induced allergic disorders associated with a Th1/Th2 immunomodulatory effect using GPMT. The results indicate that TCE can induce renal tubular cell damage through the secretion of high levels of cytokines, suggesting that TCE may result in immune system dysfunction. This novel mechanism may account for TCE-induced inflammation in chronic kidney diseases.

This study was kindly supported by a research grant from the National Natural Science Foundation of China (NSFC Grant Nos 81460470 and 81660516).

Authors declare no conflict of interest. 


\section{References}

1. Shen T, Zhu QX, Yang S, et al. (2008): Trichloroethylene induced cutaneous irritation in BALB/c hairless mice: histopathological changes and oxidative damage. Toxicology 248: 113-120.

2. Moran MJ, Zogorski JS, Squillace PJ (2007): Chlorinated solvents in groundwater of the United States. Environ Sci Technol 41: 74-81.

3. Bakke B, Stewart PA, Waters MA (2007): Uses of and exposure to trichloroethylene in U.S. industry: a systematic literature review. J Occup Environ Hyg 4: 375-390.

4. Nakajima T, Yamanoshita O, Kamijima M, et al. (2003): Generalized skin reactions in relation to trichloroethylene exposure: a review from the viewpoint of drug-metabolizing enzymes. J Occup Health 45: 8-14.

5. Khan S, Priyamvada S, Khan SA, et al. (2009): Effect of trichloroethylene (TCE) toxicity on the enzymes of carbohydrate metabolism, brush border membrane and oxidative stress in kidney and other rat tissues. Food Chem Toxicol 47: 1562-1568.

6. Xu X, Yang R, Wu N, et al. (2009): Severe hypersensitivity dermatitis and liver dysfunction induced by occupational exposure to trichloroethylene. Ind Health 47: 107-112.

7. Harth V, Bruning T, Bolt HM (2005): Renal carcinogenicity of trichloroethylene: update, mode of action, and fundamentals for occupational standard setting. Rev Environ Health 20: 103-118.

8. Jollow DJ, Bruckner JV, McMillan DC, et al. (2009): Trichloroethylene risk assessment: a review and commentary. Crit Rev Toxicol 39: 782-797.

9. Lock EA, Reed CJ (2006): Trichloroethylene: mechanisms of renal toxicity and renal cancer and relevance to risk assessment. Toxicol Sci 91: 313-331.

10. Kobayashi R, Ikemoto T, Seo M, et al. (2010): Enhancement of immediate allergic reactions by trichloroethylene ingestion via drinking water in mice. J Toxicol Sci 35: 699-707.

11. Zhang L, Bassig BA, Mora JL, et al. (2013): Alterations in serum immunoglobulin levels in workers occupationally exposed to trichloroethylene. Carcinogenesis 34: 799-802.

12. Yu JF, Leng J, Shen T, et al. (2012): Possible role of complement activation in renal impairment in trichloroethylene-sensitized guinea pigs. Toxicology 302: 172-178.

13. Cai P, Konig R, Boor PJ, et al. (2008): Chronic exposure to trichloroethene causes early onset of SLE-like disease in female MRL +/+ mice. Toxicol Appl Pharmacol 228: 68-75.

14. Jia Q, Zang D, Yi J, et al. (2012): Cytokine expression in trichloroethylene-induced hypersensitivity dermatitis: an in vivo and in vitro study. Toxicol Lett 215: 31-39.

15. Blossom SJ, Pumford NR, Gilbert KM (2004): Activation and attenuation of apoptosis of CD4+ T cells following in vivo exposure to two common environmental toxicants, trichloroacetaldehyde hydrate and trichloroacetic acid. J Autoimmun 23: 211-220.

16. Blossom SJ, Doss JC, Hennings LJ, et al. (2008): Developmental exposure to trichloroethylene promotes CD4+ T cell differentiation and hyperactivity in association with oxidative stress and neurobehavioral deficits in MRL+/+ mice. Toxicol Appl Pharmacol 231: 344-353.

17. Wang G, Wang J, Fan X, et al. (2012): Protein adducts of malondialdehyde and 4-hydroxynonenal contribute to trichloroethene-mediated autoimmunity via activating Th17 cells: dose- and time-response studies in female MRL+/+ mice. Toxicology 292: 113-122.
18. Bassig BA, Zhang L, Tang X, et al. (2013): Occupational exposure to trichloroethylene and serum concentrations of IL-6, IL-10, and TNF-alpha. Environ Mol Mutagen 54: 450-454.

19. Zhang YF, Li PM, Zhang ZM, Luo B (2013): Analysis the changes of peripheral blood routine and T lymphocyte subsets of trichloroethylene induced medicamentosa-like dermatitis patients. International Journal of Laboratory Medicine 33: 2565-2566.

20. Zhu J, Paul WE (2010): Peripheral CD4+ T-cell differentiation regulated by networks of cytokines and transcription factors. Immunol Rev 238: 247-262.

21. Mahajan SG, Mehta AA (2011): Suppression of ovalbumin-induced Th2-driven airway inflammation by beta-sitosterol in a guinea pig model of asthma. Eur J Pharmacol 650: 458-464.

22. Fernandez TD, Mayorga C, Torres MJ (2008): Cytokine and chemokine expression in the skin from patients with maculopapular exanthema to drugs. Allergy 63: 712-719.

23. Qiang Jia, Dan Zang, Juan Yi (2012): Cytokine expression in trichloroethylene-induced hypersensitivity dermatitis: An in vivo and in vitro study. Toxicology Letters 215: 31-39.

24. Guo RJ, Wang L, Shen T (2009): Changes of serum TNF- $\alpha$ and IL-1 $\beta$ levels in guinea pig sensitized with trichloroethylene. China Occupational Medicine 6: 467-469. 